Automatic Tracking of the Intersection of a Laser and Electron Beam

B.T. Turko, R.Z. Fuzesy, D.A. Pripstein, M. Kowitt, O. Chamberlain, G. Shapiro

Lawrence Berkeley Laboratory

University of California

Berkeley, CA 94720

and

E. Hughes

Stanford Linear Accelerator Center

Stanford, CA 94309

May 1990 
LBL-29017

\title{
AUTOMATIC TRACKING OF THE INTERSECTION OF A LASER AND ELECTRON BEAM
}

\author{
B.T. Turko, R.Z. Fuzesy, D.A. Pripstein, \\ M. Kowitt, O. Chamberlain, G. Shapiro (LBL) \\ and $E$. Hughes, (SIAC) \\ Lawrence Berkeley Laboratory \\ One Cyclotron Road \\ Berkeley, Calfornia 94720 U.S.A.
}

\begin{abstract}
For the Compton Polarimeter experiment at the Stanford Linear Accelerator the crossing point of a laser beam and an electron beam must be kept accurate and stable. An electronic system is described for the automatic tracking and correcting of the beam crossing. A remote CCD camera, relatively insensitive to electromagnetic disturbance, records small displacements of the pulsed laser beam. Video signals are analyzed at a remote station, the amount of drift from a selected reference point determined and the appropriate correction commands sent to the motorized mirror deflecting the laser beam. A description of the system, its performance and the test results are presented.
\end{abstract}

\section{INTRODUCTION}

The intersection point of a laser beam with an electron beam in the Compton Polarimeter Experiment [1] at SLAC must be accurately positioned and should remain so at all times. Drifts comparable only to a small fraction of the laser beam diameter ( $4 \mathrm{~mm})$ can be tolerated. An automatic electronic tracking system is described, using a charge-coupled-device (CCD) video camera for monitoring the beam crossing point in the underground experiment. Images of laser pulses seen by the camera on a reference target are transferred by a coaxial cable to a remote video display and the control circuits located in the surface trailer area.

A sketch of the experiment is shown in Fig. 1. The Spectra Physics (Model GCR-11) Laser light pulses are transmitted to the underground experimental area through pipes and are deflected by mirrors (including the motor controlled mirror) through a polarizer and then into the high vacuum electron beam pipe. In this chamber the laser beam crosses the electron beam and is directed into a detector system. A fraction ( 0.258$)$ of the laser beam passes through a partially silvered mirror and strikes a reference target, which produces an image recorded by the camera. A CCD camera [2] was selected for its relative insensitivity to electromagnetic fields present in the experimental area. The interline-transfer type of CCD sensor used in the camera is uniquely suitable for recording pulsed images because there is no need for synchronization of the camera with the laser pulses regardless of the pulse 1 ight rate. The Falrchild Weston CCD 222 sensor has sufficient resolution so that the position of the laser image can be determined within tens of microns on its $11.4 \times 8.8 \mathrm{~mm}$ sensitive area.

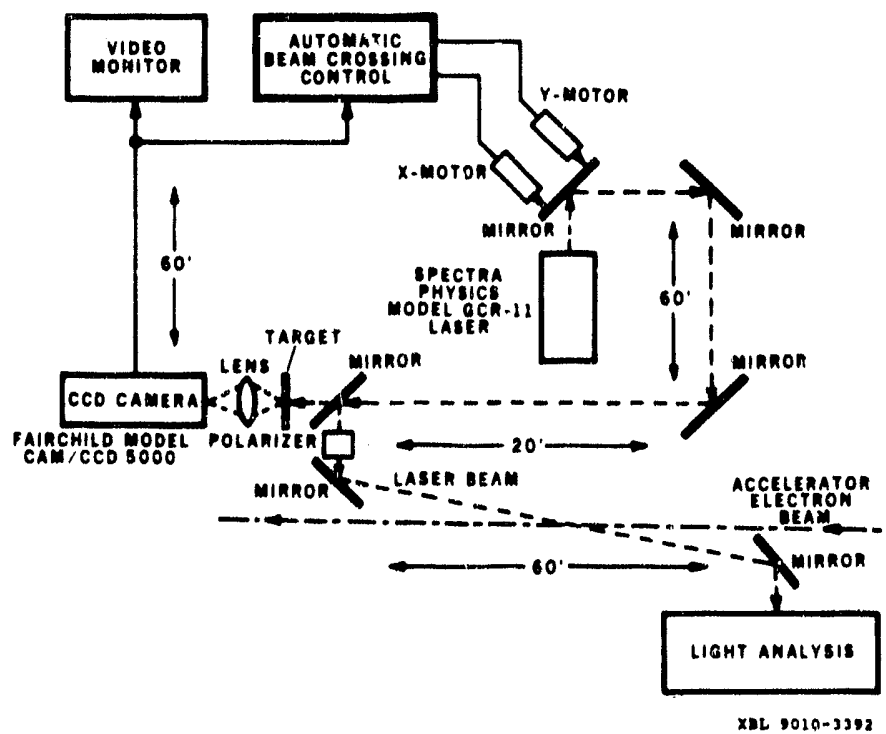

Fig. 1. Compton polarimeter experiment block diagram.

Each laser pulse image is analyzed by the electronic control circuit, described below. Any desired reference position of the image center within the video frame can be selected manually. The actual position of the image center is found by the circuit. A displacement of the image center from the reference position is determined and a pair of motors actuated to move an $X-X$ mirror stage, which directs the laser beam. The beam is deflected by the mirror until its center is moved to the reference position, (within presettable error, $\Delta X$ and $\Delta Y$ ). This error is permitted in order to prevent 
the image position correction from reacting too frequently to minor mechanical vibrations and drifts.

\section{DESCRIFTION OF OPERATION}

A block diagram of the electronic circuitry for beam tracking is shown in Fig. 2. The negative portion of the video signal from an external CCD camera provides for an oscilloscope synchronization and the posilive portion of the signal defines the intensity of the image. A Video sync. Stripper Circuit separates the timing information from the composite video signal. A level discriminator separates the useful image from the noise and the low intensity background signal from the laser beam spot image. Such an image typically appears as a pulse on several consecutive video lines (such as that in Fig. 4). A peak sensing discriminator thus defines the exact horizontal position of the laser beam image.

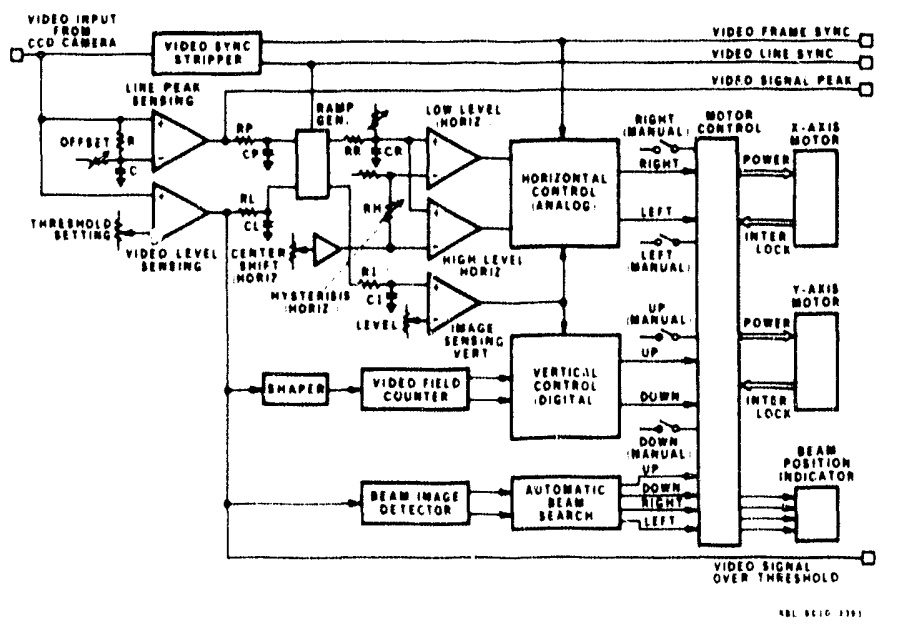

Fig. 2. Block diagram of analog and digital circuits for control and correction of laser and electron beam crossing

A horizontal control analog circuit considers the above information in order to determine the extent to which horizontal beam position is displaced from the preset reference point. A command is issued to motorized mirror gimbal to move the beam back to the reference point.

The vertical displacement of the beam image center from the preset reference position is found by the digital vertical control circuit. The vertical deflection motors are then turned on until the mirror gimbal returns the beam to the reference point.
However, the circuit reacts only if the image center drifts from the preset. reference point $\left(X_{0}, Y_{0}\right)$ by an amount greater than $\pm \Lambda X$ (right, left) or $\pm \Lambda Y$ (up, down). A corresponding beam position indicator light goes on when the beam crosses this $\pm \Delta X, \pm \Delta Y$ limits. At the same time the appropriate mirror stage motor is started to move the laser beam back to the reference point. The motors stop when the beam reaches the reference band or when the beam is completely outside the CCD camera range. Also, an interlock control stops the motors when the mechanical limits of the mirror gimbals are reached.

If the beam moves out of the CCD camera range, and the control can be set in "search mode", an automatic beam search circuit starts operating the motors, moving the laser beam by deflecting the mirror in a ziz-zag pattern. An area much larger than the CCD camera range is covered. Once the beam comes into the $C C D$ camera range, the control circuit takes over again, moving the beam center to the reference point.

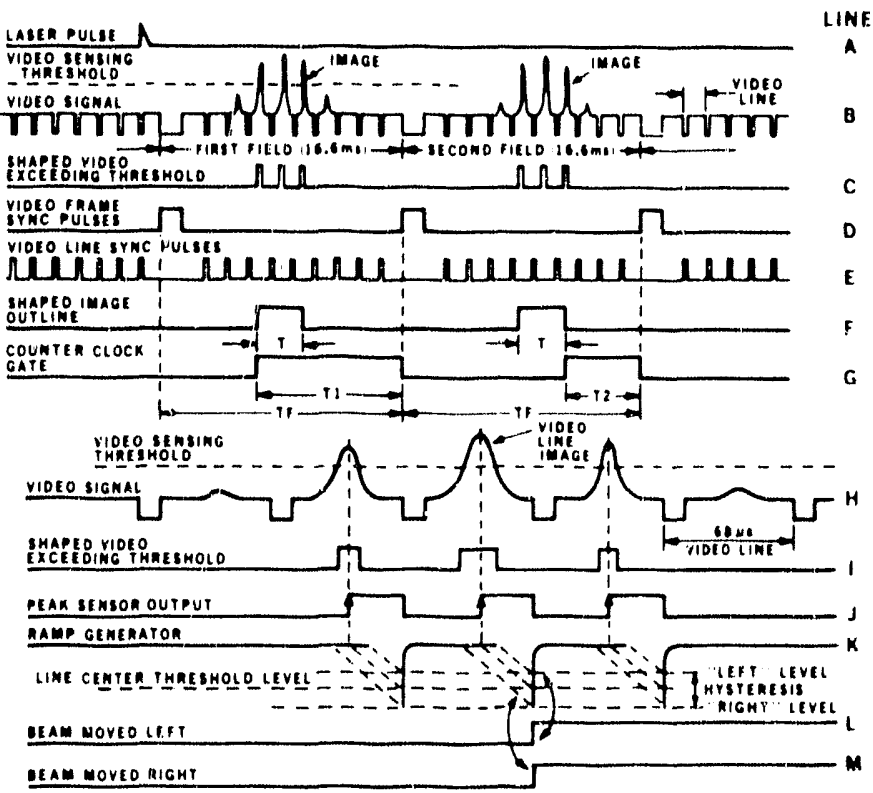

Fig. 3. Timing diagram of video signal waveforms used in defining laser beam image displacement.

Also, the motors can be operated manually at any time and the beam position verified either on the TV monitor or on the control circuit's beam position indicator.

Most of the beam tracking circuitry is analog since this permits them to operate in real time. A simplified timing diagram is shown in Fig. 3 and a typical video line scan of a beam image as seen on an oscilloscope is shown in Fig. 4. Refering 
again to Fig. 3, CCD camera video signal (Iine B) is first stripped of the trains of frame and line sync. pulses (1ines D and E). A video level discriminator separates the top of the (generally) circular beam image (line $C$ ). The peak of one line of the beam image (Fig. 4) and Fig. 3, lines $H, J$ and $K$ is sensed by a zero-crossing discriminator, defining precisely the peak's horizontal position. A ramp generator and two level discriminator control the presettable beam center reference position, $X_{0}$, and width of error band, $\pm \Delta X$.

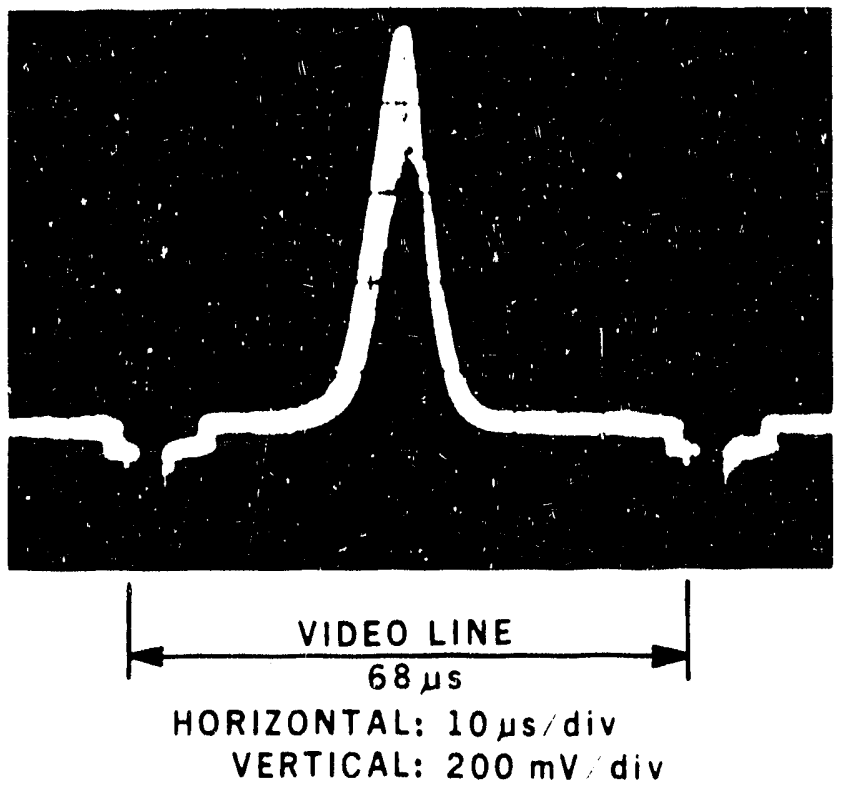

xB1 9010-8452

Fig. 4. Single video line image of a laser pulse hitting a teflon target.

The vertical position of the image is determined in part digitally. First, the width $T$ of the image is found (line F, Fig. 3 ) and the time $T_{1}$ and $T_{2}$ between the leading and the trailing edge of the two consecutive $T$ pulses and the frame's end are digitized by the video line pulses. The center of $\mathrm{T}$ is found next by a counter performing a simple $\left(\mathrm{T}_{1}+\mathrm{T}_{2}\right) / 2$ operation. The counter generates an UP or DOWN signal depending on the difference between the image center position and a digital (presettable) vertical reference $Y_{0}$. It should be noted that the video camera works in an "interlaced" mode, which produces two a djacent video fields (as in Fig. 3) for each laser pulse (Iines $A$ and $B$ ).

\section{CONCLUSIONS}

The electronic circuits for laser beam control, as described in this paper, were built and first tested in a laboratory set-up, simulating as much as possible real conditions. A $3 \mathrm{~mW} \mathrm{Ne}-\mathrm{He}$ laser beam was deflected on a teflon target. A Fairchild Weston Model CCD 5000 solid state video camera was focused on the image of diffused laser spot showing on the target. Teflon diffusion eliminated jagged multiple images normally associated with coherent light. Multiple image peaks would confuse the circuit in determining the exact coordinates of the beam position. A motor driven $X-Y$ stage with a deflecting mirror was used for returning the beam center back to the original position in order to correct for drifts expected under real conditions.

The system was also tested under pulsed light conditions resembling those expected in the Compton Polarimetex Experiment, where a Spectra Physics GCR-11 laser is being used (rate: $10 \mathrm{~Hz} ; \lambda=532 \mathrm{~nm}$; energy: $150 \mathrm{~mJ} /$ pulse; pulse width: $7.5 \mathrm{~ns}$ FWHM). The circuit analyses individual video frames and only those containing a laser beam image, regardless of the pulse rate. Also, the camera does not have to be synchronized with the laser due to the interline transfer structure of the CCD sensor in the camera.

\section{ACKNOWLEDGMENTS}

This work was performed as part of the program of the Electronics Research and Development Group, Electronics Engineering Department of the Lawrence Berkeley Laboratory, University of California, Berkeley. The work was partially supported by the U.S. Department of Energy under Contract Number DE-ACO3-76SF00098.

Reference to a company or product name does not imply approval or recommendation of the product by the University of California or the U.S. Department of Energy to the exclusion of others that may be suitable.

\section{REFERENCES}

[1] D. Blockus et al., Proposal for Polarization at SLC SLAC-Proposal-SLC-01 (1986).

(2) CCD Sensors, Systems and Development Technology, Fairchild Weston Systems Inc. Databook. 

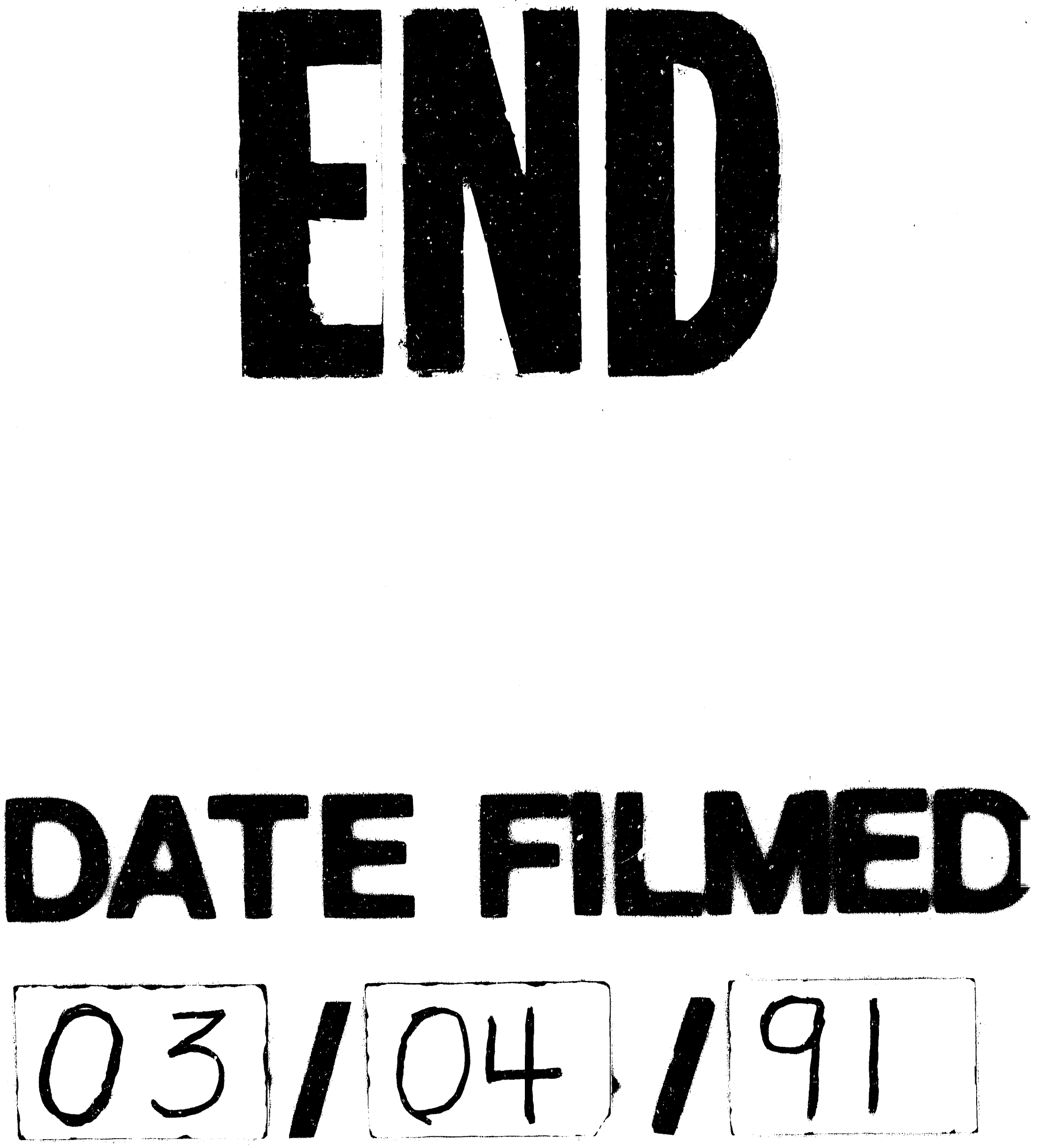
